\title{
En torno a la interpretación de la sala triabsidada del Palatium de Corduba.
}

\author{
Rafael HIDALGO PRIETO. \\ Universidad Pablo de Olavide de Sevilla
}

\section{Resumen}

En este artículo analizamos la configuración arquitectónica del triclinium triconque del palatium de Córdoba, con especial atención a la interpretación de las estructuras documentadas en su interior. Proponemos la posibilidad de que estas estructuras formaran parte de una transformación de la concepción y diseño original del triclinium durante la construcción del palatium. De esta manera, la sala triabsidada diseñada en origen se habría comenzado a construir, aunque durante el propio proceso de obra se habría sustituido por una nueva sala, en la que los ábsides se sustituyen por exedras rectangulares. La sala resultante de esta transformación responde a un original modelo arquitectónico, en cierta medida parangonable con el del triclinium del palacio de Diocleciano en Split.

Palabras clave: Cercadilla, Córdoba, tetrarquía, arquitectura palatina, trichorum.

\section{Summary}

In this paper we analyse the architectonic configuration of the triconch triclinium in the palatium of Cordoba, paying special attention to the interpretation of the documented structures inside. Our proposal is based on the possibility that these structures were part of the transformation of the first conception and design of the triclinium during its construction. Thus, the triabsidal room designed in the beginning began to be built but, in this very building process, it would have been changed by a new room in which the former absides were substituted by rectagular exedrae. The resulting room by these transformations is an original architectonic model, which could be compared in a sense to the triclicnium of the Diocletian's palace of Split.

Keywords: Cercadilla, Cordoba, tetrarchy, palatial architecture, trichorum.

Sin duda uno de los más importantes hallazgos de la arqueología española de los últimos decenios ha sido el descubrimiento del palacio de Cercadilla en Córdoba (fig. 1 y 2). El edificio, por la propia circunstancia de constituir un unicum de la arquitectura romana, junto a otras peculiaridades que lo definen, ha proporcionado un hito fundamental para entender la arquitectura palatina de época tetrárquica y la definición de buena parte de los modelos que conforman la arquitectura residencial tardoantigua.
No entraré aquí en cuestiones relativas a la datación e interpretación del palatium, sobre las que volveré en nuevas ocasiones. Aunque considero oportuno aludir al menos brevemente a ellas para precisar el ámbito en el que se debe entender e interpretar Cercadilla, en lo concerniente tanto a los modelos arquitectónicos en los que se fundamenta su diseño como a su relación con otros de la época, coincidiendo con lo ya defendido en otras ocasiones ${ }^{1}$.

1 Sobre la datación e interpretación del palatium de Córdoba, véase especialmente Hidalgo, 1996. 


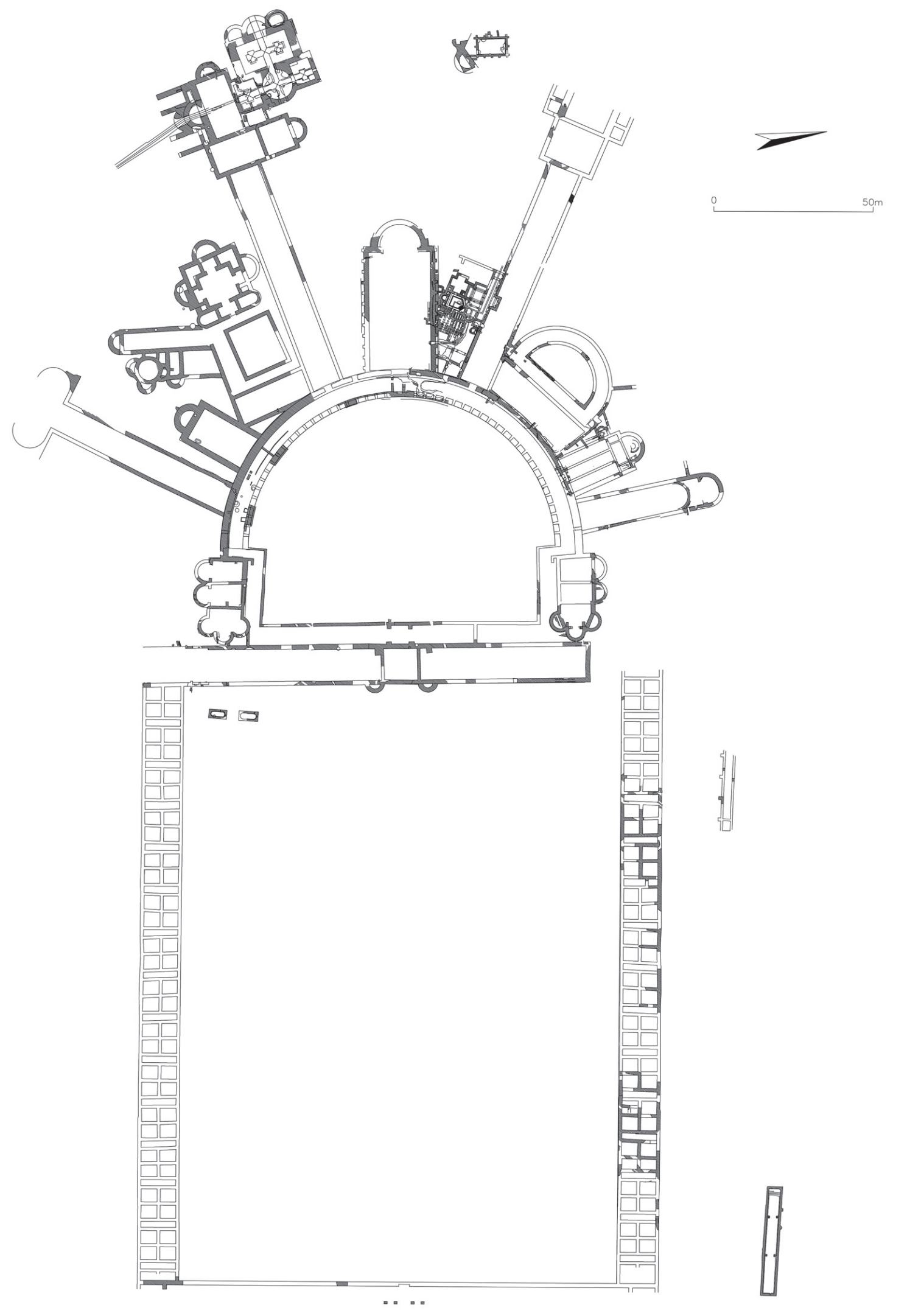

Figura 1.- Planta del palatium de Córdoba. 


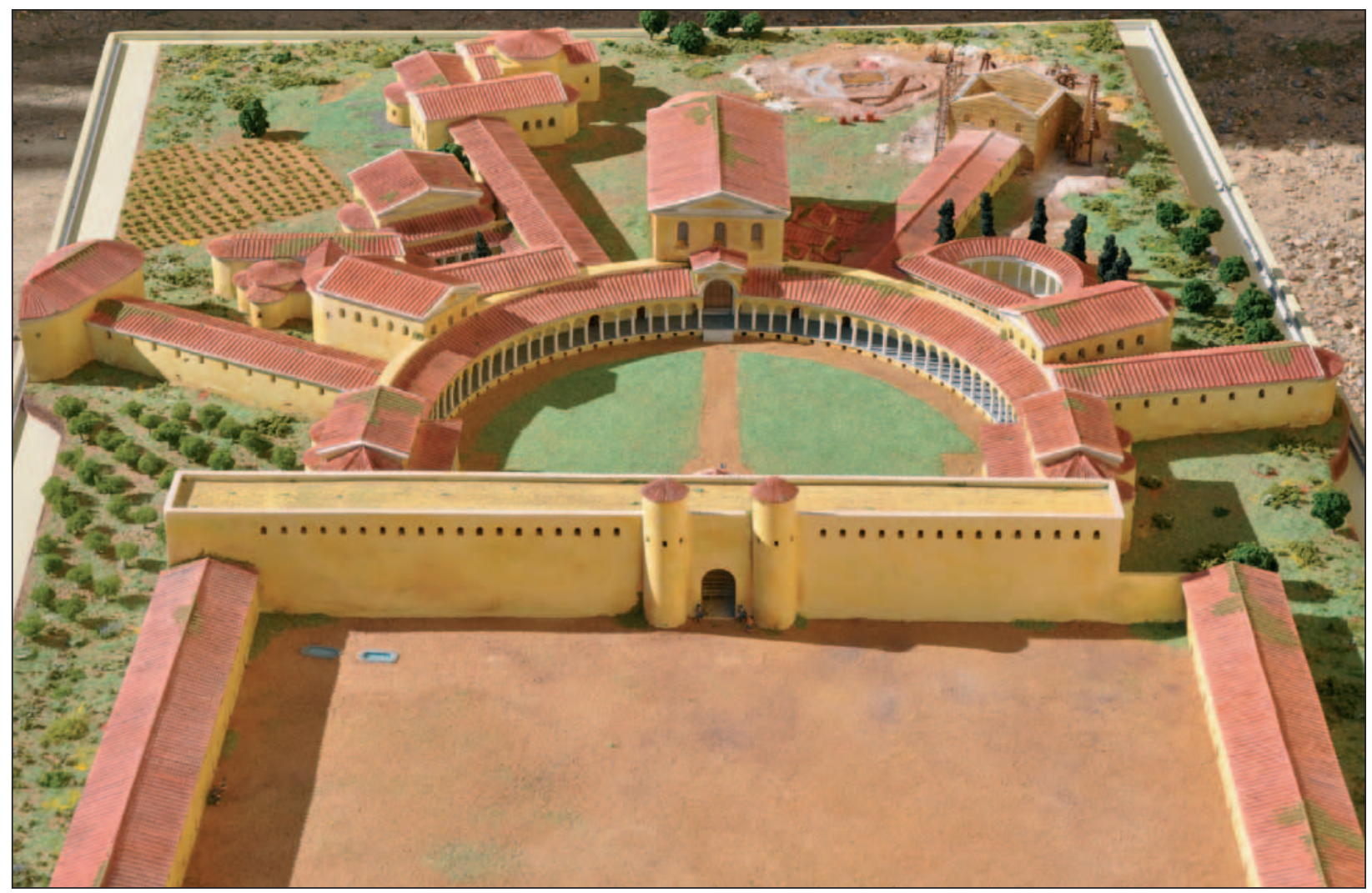

Figura 2.- Maqueta del palatium (interpretación del edificio: R. Hidalgo; ejecución de la maqueta: J.A. Ortega y equipo Escuela Taller Fernando III El Santo de Córdoba).

A efectos cronológicos no existen criterios para desechar el dato fundamental aportado por la inscripción imperial recuperada en el yacimiento (Hidalgo y Ventura, 1994 e Hidalgo, 1996, 143147). En ella, la mención de Constancio Cloro y Galerio con el título de Césares, permite afirmar que el edificio se construyó -y concluyó- durante la primera tetrarquía.

A pesar de lo parco de lo conservado en el texto, frente a la lectura hasta ahora propuesta ${ }^{2}$ no se ha planteado hasta la fecha ninguna otra posible lectura alternativa, ni se ha demostrado que la ahora imperante sea errónea, de manera que se debe seguir aceptando su validez y, con ello, su destacada incidencia en la datación del palacio cordobés. En ese mismo sentido, en lo que respecta al contexto arqueológico del que procede la inscripción, ésta se recuperó en un nivel de abandono de época tardoantigua documentado en el interior del baño central de las termas del palacio
(Hidalgo et alii, 1993, 143, Hidalgo, 1996, 143). Por las propias características del contenedor donde se encontró, situado por debajo del nivel de suelo, escapó a las intensas alteraciones postdeposicionales que sobre todo en época andalusí sufrió el yacimiento y al intenso proceso de saqueo de materiales del que fue objeto el palacio desde la Antigüedad Tardía.

En lo concerniente a la adscripción de la pieza en origen al palacio, existen argumentos que, sumados al propio contexto arqueológico del hallazgo, la hacen indudable. De ninguna manera se puede considerar que la pieza sea material de expolio traído a Cercadilla desde otra zona de la ciudad. En un momento posterior a la construcción del palacio y vinculado ya al proceso de reutilización y saqueo del edificio -como es el contexto arqueológico concerniente al hallazgo de la inscripción-, tal posibilidad carece de sentido, en tanto que realmente es el propio material del edi-

2 Hidalgo y Ventura, 1994, aceptada en los mismo términos en los que se formuló en CIL II ${ }^{2} / 7,260 \mathrm{a}$. 
ficio el que está siendo objeto de saqueo y reutilización, ya sea en nuevas construcciones llevadas a cabo en el propio yacimiento, o bien en otras edificaciones de la ciudad ${ }^{3}$. No es plausible pensar que a un edificio de tales proporciones, ya en gran parte abandonado, que está siendo saqueado y que ofrece tal volumen de material susceptible de ser reutilizado, se traiga material de acarreo de otra zona de la ciudad.

Por otro lado, tampoco es factible la posibilidad de que la inscripción formara parte del material de acarreo traído en origen para la construcción del palacio. Ese material, del que tenemos constancia en muchos casos en el monumento, ha aparecido sistemáticamente reutilizado en los muros de caementicium revestidos de vittatum mixtum que conforman el conjunto o, en un único caso, en una zona de acopio, fuera del edificio y cerca de la zona de entrada, donde se acumulaba el material que más tarde sería reutilizado como simples caementa en los muros del palacio (Fuertes e Hidalgo, 2005, 86-87; Hidalgo, 2007). Nunca ese material podría aparecer -como de hecho ha sucedido en todo lo hasta ahora excavado en el yacimiento-, de manera aislada y asociado a un contexto de abandono tardoantiguo.

En cuanto a la interpretación del palacio, la datación de su construcción durante la primera tetrarquía en combinación con los modelos arquitectónicos empleados para su diseño y construcción, sus proporciones, la técnica edilicia aplicada en su erección -completamente ajena a la tradición edilicia de la ciudad y coincidente con la empleada en los grandes conjuntos tetrárquicos-, etc., me llevan a mantener que la interpretación a todas luces más plausible obliga a vincularlo a la arquitectura palatina tetrárquica y al bien conocido proceso de descentralización de la sede imperial desde su ubicación tradicional en Roma a zonas periféricas, con la consecuente construc-

3 Sobre la posible reutilización de las columnas del palatium en la mezquita de Córdoba, véase Hidalgo, 2004, 103-104.

4 En relación con los problemas de conservación del yacimiento y con la tremenda destrucción de que fue objeto, véase especialmente Hidalgo, 1996, 15-22 e Hidalgo, 2010, 16-17.

5 Según nuestra propuesta de reconstrucción de la sala, el ción de un número nada desdeñable de palatia en todo el Imperio.

Una vez precisado el ámbito en el que se debe entender el palacio cordobés, el edificio en el que ahora centraré mi atención, la sala triabsidada, fue ya objeto de un primer estudio (Hidalgo, 1998), en el que se analizó a fondo tanto el modelo formal utilizado en su configuración como el desarrollo e incidencia de dicho modelo en la arquitectura bajoimperial. No obstante, ya en ese momento llamé la atención sobre los problemas interpretativos que presentan las estructuras que ocupan parte del interior del aula (Hidalgo, 1998, 278279), que retomo ahora con el objetivo de ofrecer una nueva alternativa para su interpretación, en parte deudora y complementaria de lo que en su momento expuse.

El edificio en cuestión (fig. 3), se encontraba en el espacio que hoy ocupa la estación de ferrocarril de Córdoba y, al igual que ocurrió con toda la superficie afectada por la superposición de la estación, fue completamente arrasado durante la construcción de ésta en $1992^{4}$. Además, todo el espacio de lo que más tarde sería la "playa de vías" de la estación, había sido ya vaciado a varios metros de profundidad con respecto al nivel previo antes del inicio de la excavación arqueológica. Como consecuencia de ello, el edificio fue destruido hasta la base de los cimientos, que en algunos tramos llegaron incluso a desaparecer, debido a que la profundidad alcanzada por tales cimientos no es regular, sino que va disminuyendo en dirección norte 5 . A tenor de tal circunstancia es evidente que la información con que contamos en relación con este sector del yacimiento es muy limitada, y la planta del edificio que hemos podido documentar, además de incompleta, pues, como ya se ha dicho, algunas de las estructuras fueron arrasadas por debajo de la base de su cimentación, corresponde en todo caso

nivel al que originalmente se encontraría el pavimento interno sería muy similar -y probablemente ligeramente superior- al del pórtico en sigma, principal distribuidor del palacio, que se encuentra a 121'30 m.s.n.m. Los cimientos del aula por nosotros excavados alcanzan la altura máxima de $118^{\prime} 60$ m.s.n.m. 


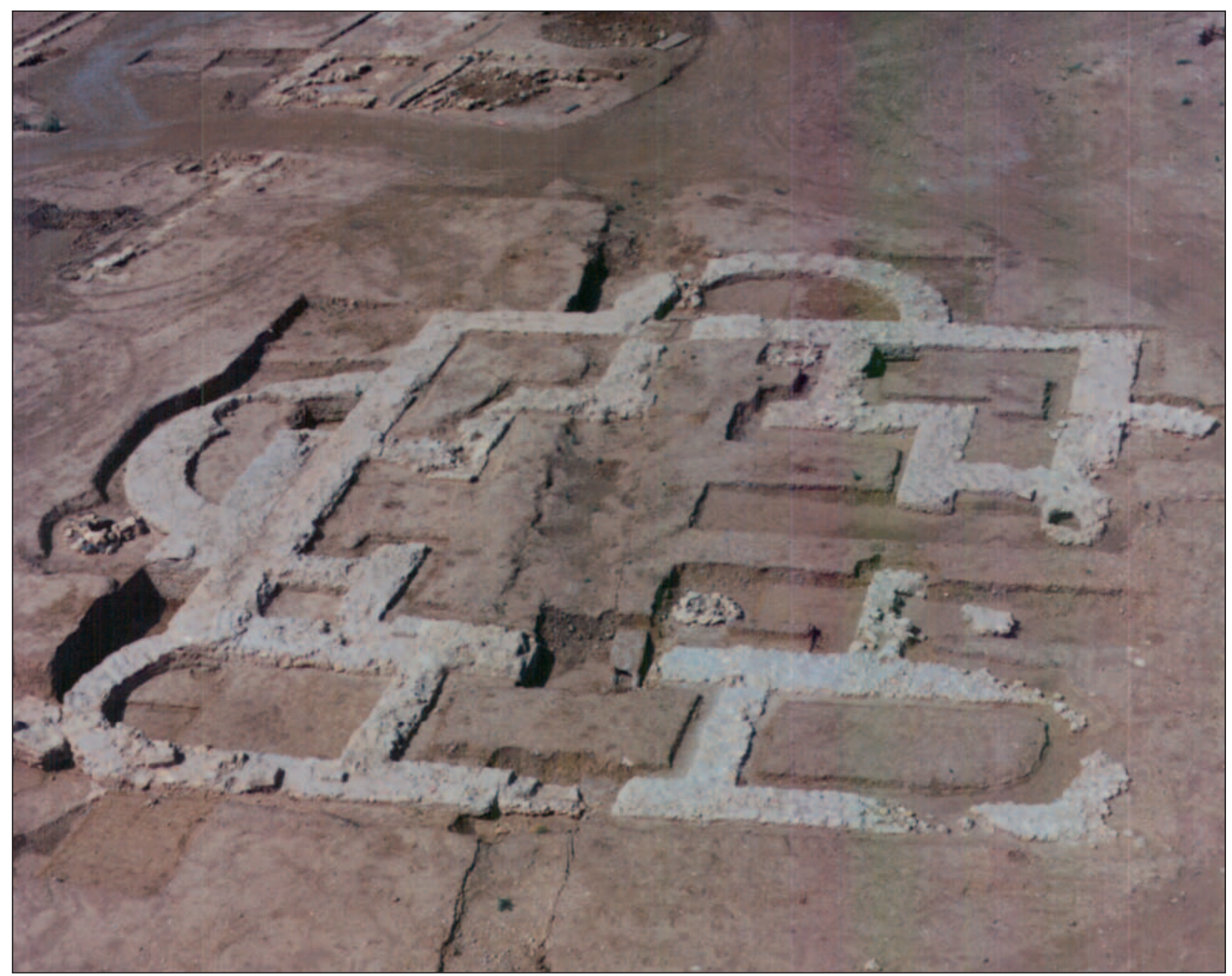

Figura 3.- Vista aérea de la triconque (desde el este).

exclusivamente a las cimentaciones. Como consecuencia de ello, evidentemente, no contamos con evidencia alguna respecto a la configuración definitiva que alcanzaría el edificio, una vez que los alzados hubieran sido replanteados y erigidos sobre los cimientos ${ }^{6}$.

En coincidencia con lo conocido en el resto del palacio (Hidalgo, 1996, 59-61), la base de la cimentación de los muros siempre apoya directamente sobre el terreno geológico y, por eso mismo, la profundidad que alcanzan las zanjas para la cimentación de los muros se redujo progresivamente hacia el norte, adaptándose al declive natural del terreno en el lugar. Como consecuencia de este proceso constructivo, como ya se ha dicho, durante la excavación de esta zona, pudimos constatar que la potencia conservada de los muros disminuía en dirección norte, hasta desaparecer. Las estructuras que conforman el lateral norte del edificio, desaparecidas antes del inicio de nuestra excavación, han sido restituidas en la planta atendiendo a criterios de axialidad, evidentes en el edificio.

Como fácilmente se puede apreciar con una mera observación de la planta, el trichorum no está aislado, sino que forma parte de un conjunto conformado por tres elementos diferentes, organizados según un mismo eje: un primer cuerpo rectangular, que funcionaría como vestíbulo de acceso, un peristilo central, concebido como espacio

6 De ello se deduce también que no existen vestigios en relación con el pavimento, estratigrafía de ocupación y abandono, etc. 
de iluminación y tránsito, que conduce, finalmente, a la sala triabsidada, en la que concluye el recorrido ${ }^{7}$.

El eje de estos tres elementos no se dispone de forma radial con respecto al centro del pórtico en sigma, como es norma en la mayoría de los edificios que se disponen en su entorno, sino que lo hace en relación con el pasillo situado inmediatamente al norte, al que se adosa. Tal circunstancia viene motivada por el hecho de que el acceso a este sector no se llevaría a cabo directamente desde el pórtico en sigma, sino que se haría indirectamente desde el pasillo inmediato, que sí se encuentra conectado con el pórtico en sigma. De esta forma, desde el mencionado pasillo se accedería a la primera sala rectangular que funcionaría como vestíbulo, desde donde se alcanza la triconque atravesando el peristilo. Ello se debe poner en relación con la jerarquización de espacios $-y$ de sus correspondientes circuitos de acceso y tránsito-, que se hace patente en el palacio a partir de la función prevista para los distintos edificios que lo conforman. De esta manera, son los edificios de mayor carácter representativo, entre los que se deben incluir la gran sala de representación basilical que preside el eje del palacio y las dos basílicas menores, los que se sitúan en comunicación directa con el pórtico en sigma. Por su parte, las salas que desempeñan una función intermedia, entre las que se deben incluir los triclinia, se disponen, como es el caso que aquí nos ocupa, en conexión indirecta con el pórtico en sigma, mientras que, finalmente, aquellos espacios de mayor carácter privado se ubican en una posición más alejada del pórtico en sigma o con un acceso más restringido.

Del peristilo central que precede la sala triabsidada, que alcanzaría en torno a $222^{\prime} 5 \mathrm{~m}$. por 23 m., hemos podido documentar sólo los laterales y el extremo sur. Tanto el extremo norte como la zona central la hemos podido reconstruir a partir

7 Desde el peristilo y a través de un pasillo lateral, se alcanza un edificio de planta centrada, que estructuralmente se debe considerar independiente del conjunto que aquí nos interesa.

8

Sobre la información con que contamos a esos efectos y su aplicación en la propuesta de reconstrucción del peristilo, véase especialmente Hidalgo, 1998, 276; 1997, fig. 96 e Hidalgo et alii, 1996, fig. 13 y 33. de fuentes indirectas ${ }^{8}$, gracias a las cuales se puede plantear que el límite norte del patio estaría conformado por el muro que delimita el pasillo situado al norte y que el patio contaría en el centro con un peristilo, dotándose de pórtico en todos sus lados.

En lo que se refiere a la triconque, como ya se ha dicho, por su estado de arrasamiento tan sólo se ha podido documentar el caementicium de sus cimientos (fig. 4). No obstante, al menos en parte se conservan, sobre la superficie del caementicium, débiles vestigios de lo que pudo ser la huella de la hilada de sillares que, siguiendo la secuencia constructiva reproducida en muchas otras zonas del palacio, constituía el final del cimiento y sobre la que se replanteaba el alzado de los muros, ya con sus dimensiones y trazado precisos $^{9}$. Aunque en muchos casos los errores o, mejor, las imprecisiones de las cimentaciones se corregían mediante el replanteo de los alzados, en esto caso se perciben ciertas deformaciones que no habrían podido ser corregidas en los alzados. En concreto, los muros de los laterales se desvían más de un grado con respecto a los que deberían intectar con ellos perpendicularmente.

Por su parte, el ingreso al interior del aula se llevaba a cabo a partir de un vestíbulo flanqueado por sendos ábsides en sus extremos. El vano, del que en este caso también se conservaba sólo la cimentación, en la que apenas supera los dos metros de anchura, se encuentra ligeramente desplazado con respecto al eje de axialidad. Probablemente esta circunstancia se corregiría en el alzado, del mismo modo que muy probablemente en alzado se dotaría también de mayor anchura.

En el interior, la sala alcanza 16'3 m. de longitud frente a 14' $1 \mathrm{~m}$. de anchura y los ábsides de sus laterales en torno a 6'7 m. de diámetro. Además, en ese espacio interior se incorporan

9 A la hora de ejecutar los pavimentos, se nivelaban los espacios mediante el aporte de sedimento hasta alcanzar la altura prevista para el suelo y, como consecuencia de este mismo proceso, finalmente parte del muro en alzado quedaba oculto bajo el pavimento. 


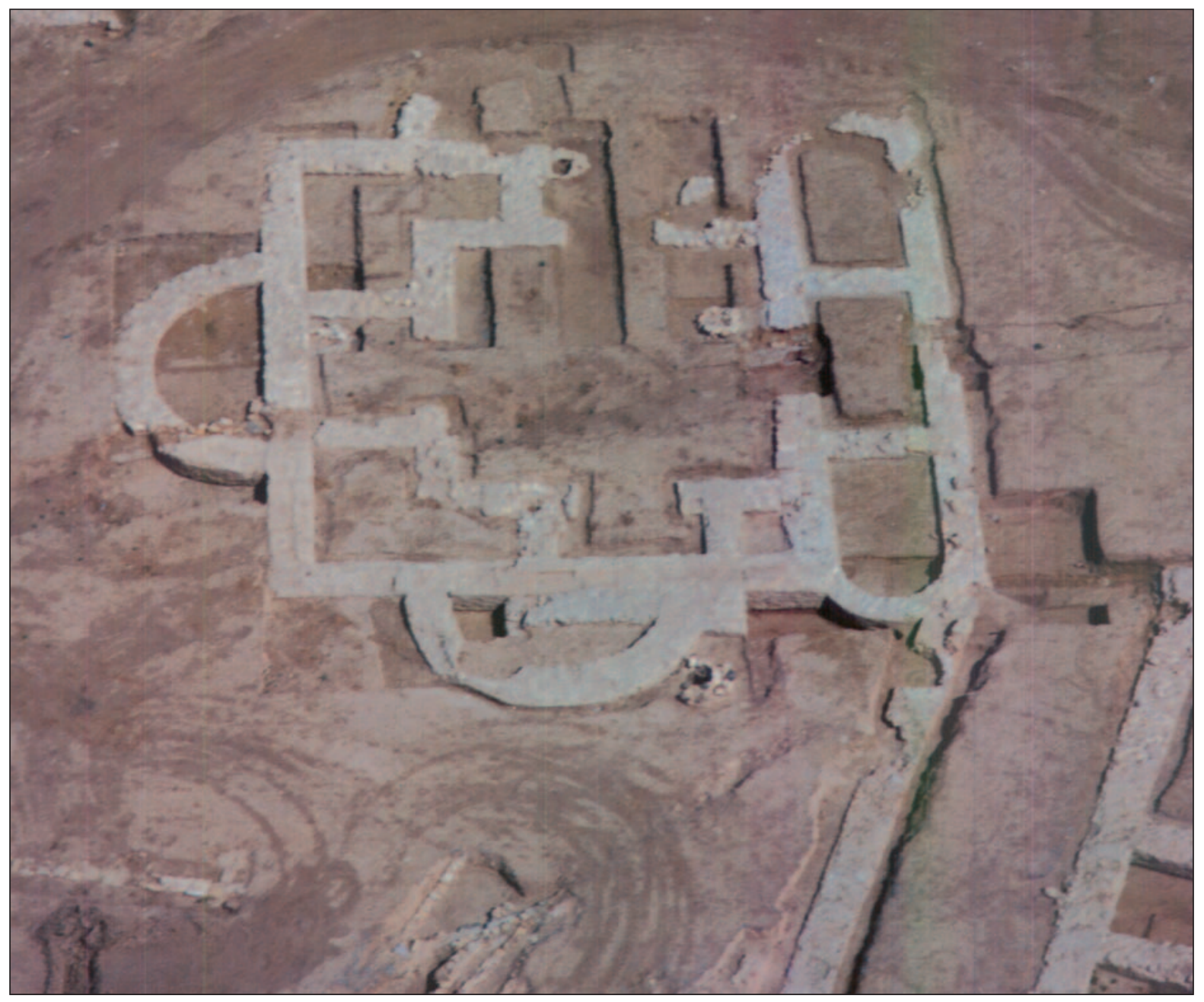

Figura 4.- Vista aérea de la triconque (desde el sur).

varios muros que, como ya se ha dicho, constituyen el objeto específico de nuestra atención aquí (fig. 5a). En ellos se observa, como primera peculiaridad, que se disponen un una posición ligeramente excéntrica con respecto al propio eje de la sala en la que se inscriben. Las estructuras en cuestión, cuentan con un primer espacio cuadrangular central, que adquiere unas dimensiones máximas internas de en torno a $10 \mathrm{~m}$. de lado, con vanos abiertos en todos sus lados, que alcanzan algo más $3 \mathrm{~m}$ de anchura. Este espacio central presenta en tres de sus lados, coincidiendo con los vanos, sendos muros pareados que conectan el espacio cuadrangular central con el perímetro de la triconque. Es importante, de cara a la ulterior interpretación de estas construcciones, llamar la atención sobre la incorporación, al sur del muro que cierra en ese mismo flanco la triconque, de un muro, en parte al exterior de la sala y adosado a un lado y otro del arranque del ábside de ese late- ral, que coincide en su trazado con los muros que conectan con el rectángulo central. Este muro, de difícil interpretación en relación con la aparente configuración final de la triconque, es un elemento fundamental para comprender esta complicada red de muros distribuidos en el interior del aula.

Dentro del proceso constructivo, es evidente que en un primer momento se construyen las estructuras maestras de la sala triabsidada, mientras que estos otros se entregan a los primeros. No obstante, en relación con estas estructuras, la primera cuestión que se debe tener en cuenta es que corresponden sin duda al proceso de construcción original del palacio. Argumento en ese sentido es en primer lugar la total coincidencia del caementicium con el que se llevan a cabo con el que se utiliza en el resto de las construcciones originales del monumento, diferenciándose en composición, color, compactación, etc., con el correspondiente a las fases de transformación tardoantiguas hasta 


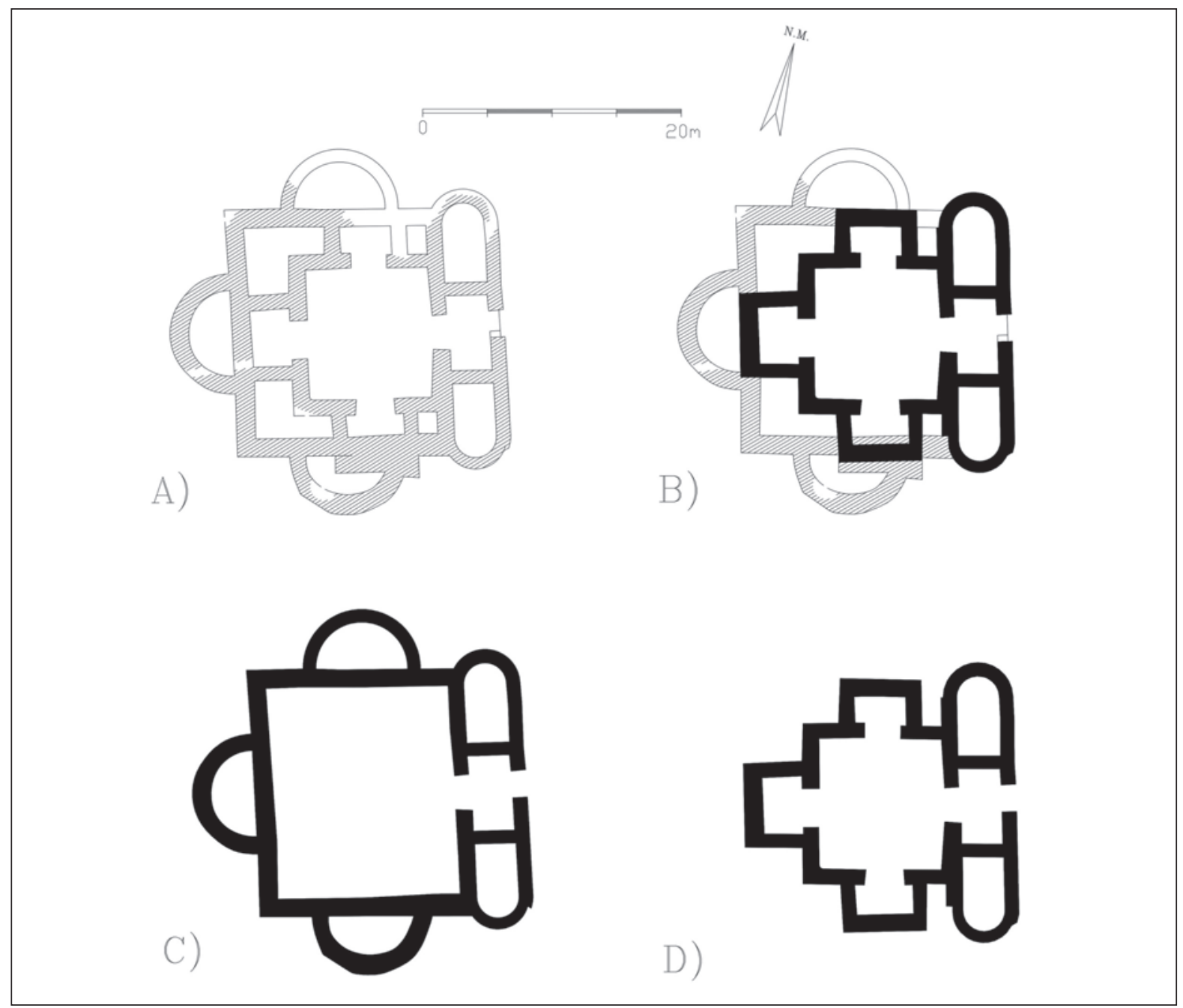

Figura 5.- Hipótesis de posible transformación en obra de la triconque. A- Planta de todas las estructuras documentadas.

B- Interpretación de las transformaciones del proceso inicial del proyecto. C- Proyecto inicial. D- Hipótesis de posible configuración de la planta final (en cimientos).

ahora detectadas en el complejo. Junto a ello, lo que es más importante, el sistema constructivo con el que se ejecutan estas cimentaciones coincide con el aplicado en el resto del aula, de manera que como ocurre con el resto de las estructuras, éstas también descienden de manera progresiva de norte a sur, buscando siempre el asiento en la zona más resistente del firme geológico. De este modo, coincidiendo igualmente con lo que sucede con el resto de las estructuras de la sala, éstas también desaparecen, hasta la base de los cimientos, al aproximarnos al lateral norte. Algo similar se puede decir en relación con el grosor que alcanzan estos cimientos, en torno a 1'2-1'3 m., coincidiendo a su vez en esto con el resto de las estructuras de la sala y, con ello, con las que conforman la construcción original del palacio.
En definitiva, todo lo visto nos lleva a plantear que no existe argumento alguno que permita considerar la posibilidad de que estas construcciones puedan corresponder a una transformación posterior del edificio, sino que, más bien al contrario, se debe pensar que todos los indicios apuntan a que corresponden a la construcción inicial y coetánea de todo el palacio dentro, claro está, del lógico proceso edilicio que conlleva la edificación de un complejo de las proporciones de Cercadilla.

Aunque, como consecuencia del estado en que se encontraba el edificio cuando llevamos a cabo su excavación, es bien difícil discernir cuál pudo ser la función de las complicadas estructuras que se distribuyen en su interior, en parte alcanzando su exterior, al menos hemos podido formular algunas hipótesis sobre su interpretación (Hidalgo, 1998, 278). 
En ese sentido, una primera posibilidad que a cualquier especialista puede venir en mente tras una mera observación de la planta, es que esta red de muros asimétricos, tanto por su propia distribución como por su asimetría con respecto a la sala que los acoge, correspondiera a un momento posterior, a una fase posterior de la vida del edificio en la que se habría alterado completamente su planta, creando un nuevo edificio. No obstante, esta hipótesis debe ser completamente rechazada en atención a lo antes dicho. Esto es, tanto la semejanza de la edilicia con la obra original, como los detalles constructivos -ya sea la profundidad descendente de los cimientos, la anchura de los muros, el encofrado directamente en zanja, etc.-, permiten claramente identificar estas estructuras con cualquier otra de las construidas para la erección del palacio. Igualmente, las pequeñas transformaciones que se producen en la adaptación del palacio a su nuevo uso cristiano durante la Antigüedad Tardía, no tienen nada que ver con las construcciones originales $\mathrm{ni}$, con ello, con estos muros. Son estructuras de escasa entidad, hechas bien con sillería o con un camementicium de escasa consistencia, utilizado además en muros que en este momento alcanzan un reducido grosor. Además, estas transformaciones tardoantiguas -las únicas que se producen en el palacio tras su construcción-, constituyen pequeñas adaptaciones o añadidos en los edificios, y no una transformación substancial, como se puede deducir de la presente en la trichora, a partir de lo que se infiere de su planta y de la entidad y profundidad de los cimientos que la conforman.

Descartada pues esa opción, una segunda posibilidad a tener en cuenta es que se tratara de la cimentación de una columnata, que habría contribuido a la sustentación de la cubierta del edificio que, en ese caso, sería más lógico suponer que hubiera estado conformada por una cúpula, mejor que por una más ortodoxa cubierta adintelada, para la que, en atención a la luz interna de la sala, no habría sido necesaria la incorporación de esas hipotéticas columnas. La distribución asimétrica de tales cimientos podría radicar en que, garanti-

10 Sobre el modelo triconque y su desarrollo véase especialmente Ebert, 1864; Deichmann, 1950; Bettini, 1955, Lavin, 1962; Duval y Cintas, 1973; Carandini, Rici y de Vos, 1982; zada su presencia en los puntos en los que deberían apoyar las columnas, no habría importado demasiado la posición en exacta simetría de sus cimientos, que evidentemente no tendrían desarrollo en alzado. Sin embargo, lo cierto es que esta posibilidad cuenta con serios inconvenientes, en el sentido de que es difícil plantear una cubierta con cúpula, al menos sobre cuatro apoyos columnados en el interior de la sala, cuya posición nunca podría haber estado completamente centrada con respecto al interior del edificio. A ello se debe sumar que, en la línea de lo antes dicho, para una sala de tales características, tanto por el modelo reproducido ${ }^{10}$ como por su luz interior, es más que lógico presumir que la cubierta se hubiera resuelto mediante la incorporación de una techumbre adintelada.

Una tercera posibilidad, sobre la que aquí quiero hacer especial hincapié, es que estas estructuras no correspondan realmente a una segunda fase, sino a una transformación de la obra durante el proceso constructivo. De esta manera, inicialmente el edificio se habría concebido como un trichorum ortodoxo, si bien, durante el proceso de ejecución, se habría decidido abandonar el diseño original y sustituirlo por el que conforman por sí mismos los muros documentados en el interior. En este proceso se habrían abandonado -sin llegar a construirse en alzado- aquellas estructuras innecesarias en el nuevo proyecto, y se habrían reutilizado, total o parcialmente, las susceptibles de serlo. Así pues, abstrayendo el diseño resultante de tal proceso (fig. $5 \mathrm{~b}$ y d), lo que efectivamente se habría construido es una sala prácticamente cuadrangular, de $10 \mathrm{~m}$. de anchura por 9' $5 \mathrm{~m}$. de longitud. Por su parte, esta sala contaría en su perímetro con tres exedras, de dimensiones también más reducidas que los ábsides del trichorum inicial. La exedra de la cabecera alcanzaría 4'5 m. de anchura por $3 \mathrm{~m}$. de profundidad; por su parte, la del lateral norte alcanzaría $4 \mathrm{~m}$. de anchura por 2'25 m. de profundidad; mientras que, por último, en lo concerniente a la situada al sur, en esta otra la anchura, coincidien-

Cambi, 1984; Grossmann, 1992; Downey, 1993; Hidalgo, 1998; Bowden y Hodges, 2011 y Verzone, 2011, 31-41. 
do con la opuesta, sería de 4 m., si bien, debido al ligero desplazamiento de la nueva sala hacia el sur con respecto a la originalmente diseñada, su profundidad se limitaría a 1'6 m. Probablemente el hecho de que la profundidad en este caso fuera excesivamente reducida, fue lo que motivó que se construyera un nuevo muro, adosado al cierre original del aula, en parte dentro de uno de los ábsides y en parte en el exterior, pero, lo que abunda aún más en esta propuesta, coincidiendo con los cierres laterales de la nueva exedra. Con este refuerzo, a la hora de replantear los alzados -evidentemente a una altura superior a la conservada-, esta exedra se habría podido replantear coincidiendo en profundidad con la opuesta, gracias a la incorporación del nuevo muro exterior, imprescindible para tal función. De esa manera, el muro en alzado habría apoyado en parte en el muro original y en parte en el nuevo refuerzo construido al efecto. En la reconstrucción de cómo debió ser la exedra sur, es de interés resaltar que la prolongación de las caras externas de los muros construidos en el interior de la triconque, que constituirían los laterales de la exedra, coinciden perfectamente con los laterales del muro adosado al exterior de la triconque, lo que viene a incidir aún más en la coherencia constructiva sin duda existente entre todas estas estructuras. A ello se une también la circunstancia, nada desdeñable, de que esta es la más plausible posibilidad que se puede argumentar para explicar e interpretar la atípica posición del refuerzo exterior con respecto al aula original.

De ser cierta esta transformación del proyecto inicial, queda por discernir qué habría ocurrido con la planta triconque ortodoxa que se habría comenzado a construir y que se habría abandonado, muy probablemente antes de llevar a cabo el replanteo de sus alzados. Esto es, ¿es posible que,

11 Sobre la difusión de la sala trilobulada en la arquitectura residencial, véase especialmente Fernández Castro, 1982, 207-208; Duval, 1987, 486-487; Rossiter, 1991, 199-213; Morvillez, 1995,15-26; Hidalgo, 1998, 281-288; Balmelle, 2001, 164-165 y 172-173; Baldini Lippolis, 2001, 60; Sfameni, 2006, 96-101. La función triclinar es en la mayoría de los casos interpretativa, como consecuencia de la adaptación del espacio arquitectónico a la nueva mensa de banquete ampliamente difundida desde el Bajo Imperio, el stibadium. De esa manera, cada ábside desempeñaría la función de acoger un stibadium, con una clara preponderancia aunque la estructura cambiara interiormente, exteriormente se mantuviera la apariencia triabsidada original? Aunque no contamos con evidencias para responder con certeza a esa pregunta y aunque no se puede descartar que así fuera, lo más lógico es suponer que no fue así, sino que la transformación del proyecto, por su entidad, debería haber afectado radicalmente a la conformación en alzado del edificio, tanto interiormente como exteriormente. A ello se debe añadir que el palacio no está concebido para ser visto desde el exterior en esta zona trasera, de modo que no parece muy lógico el esfuerzo de mantener un espacio externo sin función arquitectónica que no sería visto. Definitiva en este sentido es la superposición de la exedra sur sobre el ábside inicial, que hace prácticamente incompatible la pervivencia en alzado de las dos formas. Así pues, lo más presumible es que la triconque original quedara en una estructura en cimentación, que nunca habría contado con alzado y que habría quedado oculta bajo el nivel de suelo y, siempre, al exterior del nuevo edificio.

En lo que se refiere al esquema arquitectónico aplicado a la conformación del aula, si bien la concepción original corresponde a un modelo bien conocido en la arquitectura romana, la nueva planta que durante el propio proceso de obra pudo haber adoptado responde a un modelo sin duda mucho más singular.

Es bien sabido que la planta triabsidada constituye un esquema desarrollado con gran profusión especialmente durante la Antigüedad Tardía, constituyendo uno de los espacios de prestigio y representación más destacados de la arquitectura residencial de la época, especialmente indicado para las funciones de representación, incluidas entre éstas, claro está, el banquete ${ }^{11}$, y en muchas ocasiones en combinación con la más frecuente

del situado en el centro con respecto a los laterales (Bek, 1983, 83), y dejando el espacio central libre tanto para el tránsito del servicio que asiste el banquete como para las actividades que lo amenizaban. En el denominado "Palacio de Teodorico" en Rávena, la función de la sala triconque como triclinium queda atestiguada en una inscripción musiva presente en la propia sala (vid. Setis, 1975, 897 y Rizzardi, 1996). También evidente en ese sentido es el caso de Dougga, donde la sala triconque se dispone junto a una estancia identificada como la cocina (Poinnsot, 1958; Baldini Lippolis, 2001, 60). 
aún aula de planta absidada ${ }^{12}$. Desde la conformación en sus primeros pasos del modelo hasta su amplia difusión tardoantigua, esta planta se adapta a distintas funciones, como son las de mausoleo, ninfeo o a diversos usos dentro de la arquitectura termal (vid. Hidalgo, 1998, 281), si bien su mayor difusión, tanto por la cantidad de aulas, su variabilidad y su distribución geográfica, es la que la vincula a la arquitectura palatina y residen$\mathrm{cial}^{13}$, en la que cuenta con una gran presencia en casas y villas, muy especialmente occidentales, entre las que también se hacen presente las hispanas, y con una gran diversidad de tamaño, alcanzado desde los $50 \mathrm{~m}^{2}$ hasta casi los $300 \mathrm{~m}^{2}$ (Rossiter, 1991, 20) (fig. 6).

Por su parte, la planta en la que las exedras son rectangulares o cuadrangulares, denominada por C. Balmelle (2001, 165 y 172-173) "plan en $\mathrm{T}$ ", responde a un modelo mucho menos difundido y reproducido. Mientras que en la arquitectura termal se hace muy presente, en lo que F. Yegül (1992, 409-410) denominó “caldaria

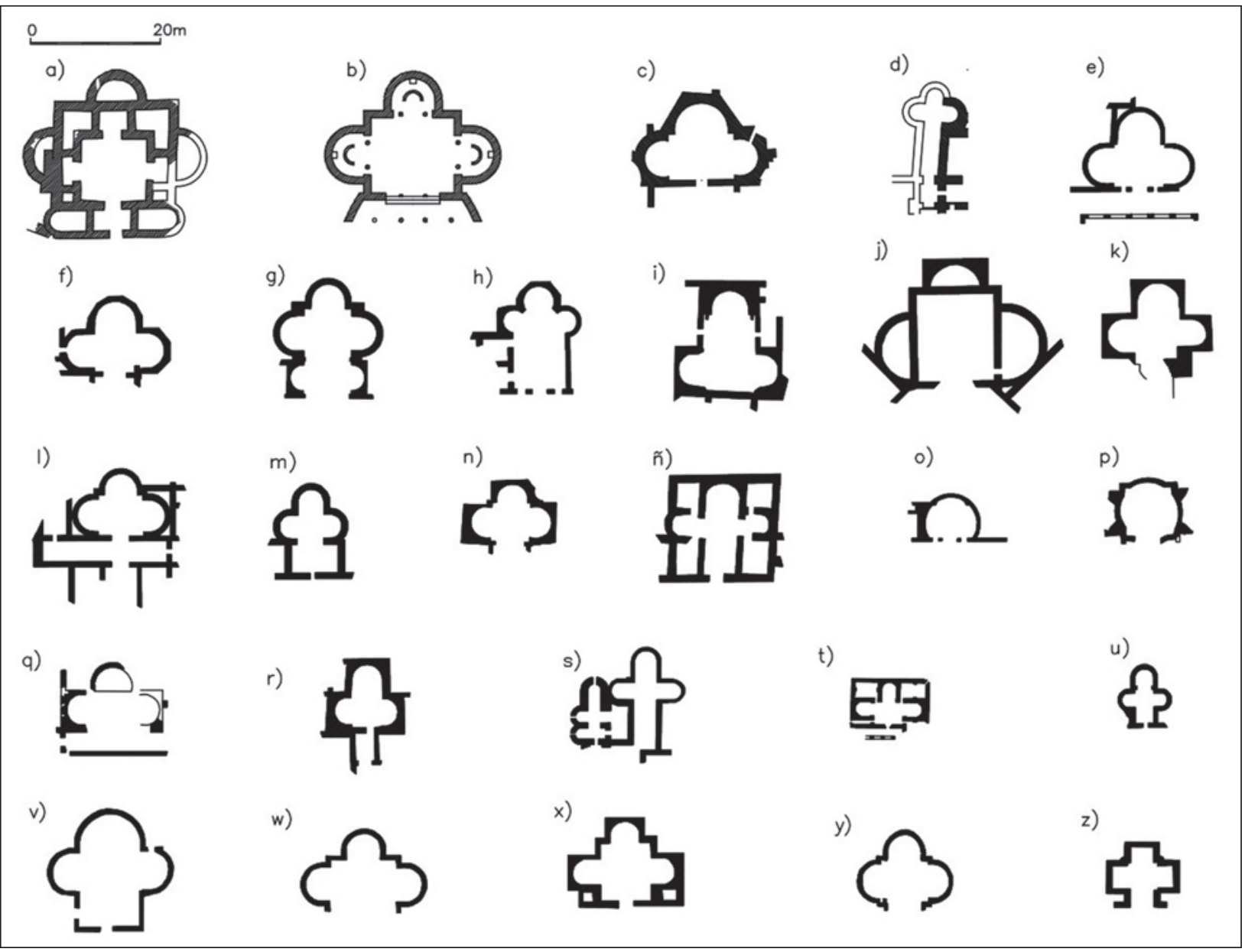

Figura 6.- Planta comparativa aulas triconques:

a) Cercadilla; b) Piazza Armerina; c) "Palacio del Gobernador" de Afrodisias; d) "Palacio del Dux" de Dura Europos; e) Villa de Patti Marina; f) "Palacio de Teodorico" en Rávena; g) Villa de Desenzano del Garda; h) Casa del Carro de Venus (Thuburbo Majus); i) Casa del Triconchos, Ptolemais; j) y k) Villa de Rabaçal; 1) Villa de las Mezquitillas, Ecija; m) Villa de los Quintanares; n)Villa de Torre Aguila; ñ) Villa de Torre de Palma; o) Villa de Almenara de Adaja; p) Villa de Carranque; q) Schola de Cartago; r) Villa de Thuburbo Majus; s) Villa de El-Alia; t) Palacio de Bosra; u) Villa del Camino Viejo de las Sepulturas, Balazote; v) Villa de Loupian; w) Villa de Quinta das Longas;

x) Villa de Blanzy-lès-Fismes; y) Villa de Cazzanello; z) Villa de Jurançon, Pont d'Oly.

12 En relación con la combinación de las aulas de planta basi-
lical con las triconques véase especialmente Scagliarini
Corlaità, 2003,166-167.
13 De ahí se difundirá su uso a martyria y, especialmente, igle- sias, con una amplísima difusión, sobre todo entre los siglos V y VI, también en esta otra función (vid. entre otros, Hidalgo, 1998, 282). 
con brazos proyectados", sin embargo, en la arquitectura residencial y representativa su presencia es escasa. Además, en ese esquema habitualmente las exedras constituyen una proyección de todo el lateral del aula y no de un pequeño tramo central, como habría ocurrido en el caso cordobés.

Cuando se aplican exedras rectangulares o cuadrangulares el esquema aparece con la misma frecuencia o incluso más como tetraconque que como triconque. En este sentido, es posible que en el edificio cordobés el aula se hubiera configurado incluso como aula tetraconque, de manera que los muros que cierran perpendicularmente el vestíbulo biabsidado que precede el aula, podrían corresponder también a la transformación de la sala y conformar, en lo que respecta a la percepción interior de los espacios, la cuarta exedra de la estancia, que funcionaría, como es habitual en tales casos, como acceso.

A efectos formales, sin duda el ejemplo de mayor interés para comparar el aula que aquí nos ocupa es el del palacio de Diocleciano en Split, que presenta en muchos aspectos concomitancias con el palacio cordobés (Hidalgo, 1997) (fig. 7). En el palacio dálmata contamos también con un triclinium triconque, en el que tampoco comparecen los ábsides perimetrales. En este caso se sustituyen por tres espacios en los que se trasciende del concepto de exedra en sentido estricto para generar tres pequeñas salas de planta cuadrada.
No obstante, aunque en Split los espacios perimetrales alcanzan unas proporciones claramente superiores a Cercadilla, el central adquiere unas proporciones muy similares en uno y otro caso.

Por las dimensiones que alcanzarían la sala y las exedras anejas según el nuevo esquema triconque propuesto, es evidente que en el edificio cordobés el espacio a utilizar para el banquete no serían las exedras, demasiado limitadas en proporciones para este fin, sino que en ese caso sería el espacio central el reservado para tal función. Ello entronca directamente con el inicio de la formalización del modelo en sus primeros exponentes, el triclinium de la Domus Flavia y el "Tre Esedre" de Villa Adriana (Hidalgo, 1998, 284), en los que, igualmente, el espacio destinado al banquete era el central, mientras que las exedras laterales desempeñaban una función complementaria.

A favor de la posibilidad de que el aula se hubiera transformado durante el proceso constructivo, es de interés tener en cuenta que éste no sería el único caso en el palacio en el que se ha documentado una transformación del proyecto, de importancia, que conlleva el abandono de una construcción ya iniciada para ser sustituida por otra. Especial mención en ese sentido merece el gran estanque ornamental documentado en la plaza que antecede al palacio (fig. 8 y 9). En un primer momento, en esta plaza se proyecta y comienza a construir un gran estanque, que alcanza unas dimensiones internas de $88^{\prime} 6 \mathrm{~m}$. de lon-
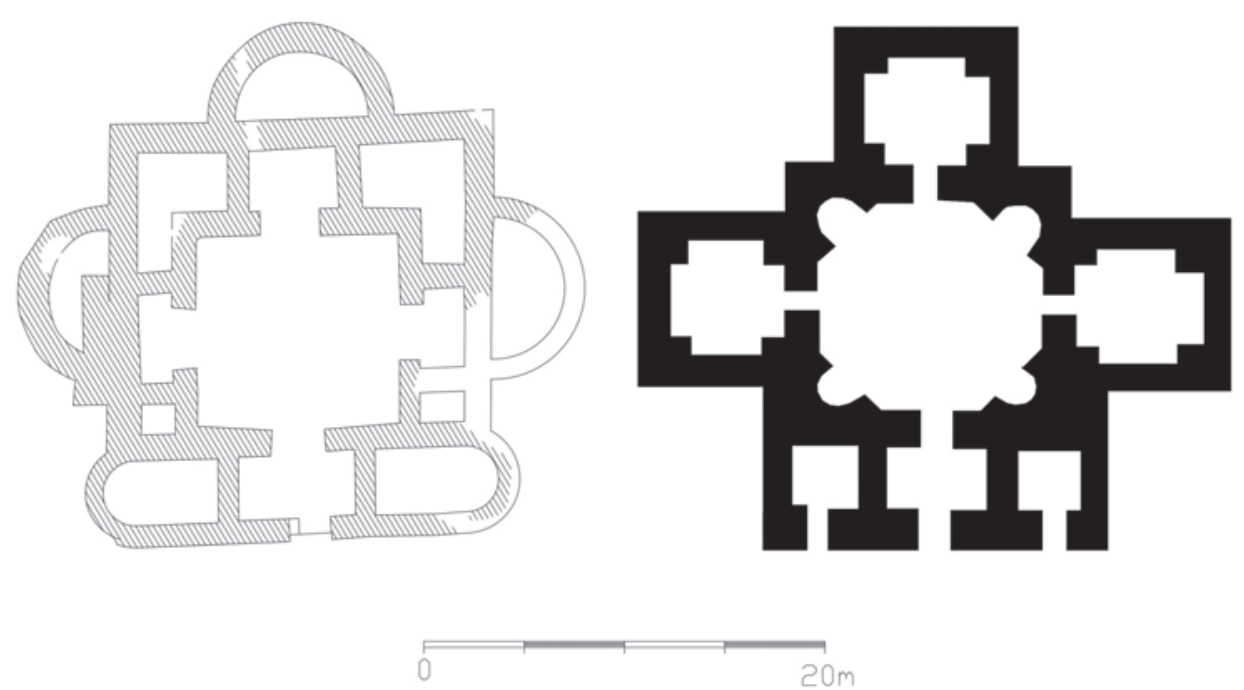

Figura 7.- Planta comparativa de las aulas triconques de Cercadilla (izquierda) y Split (derecha). 


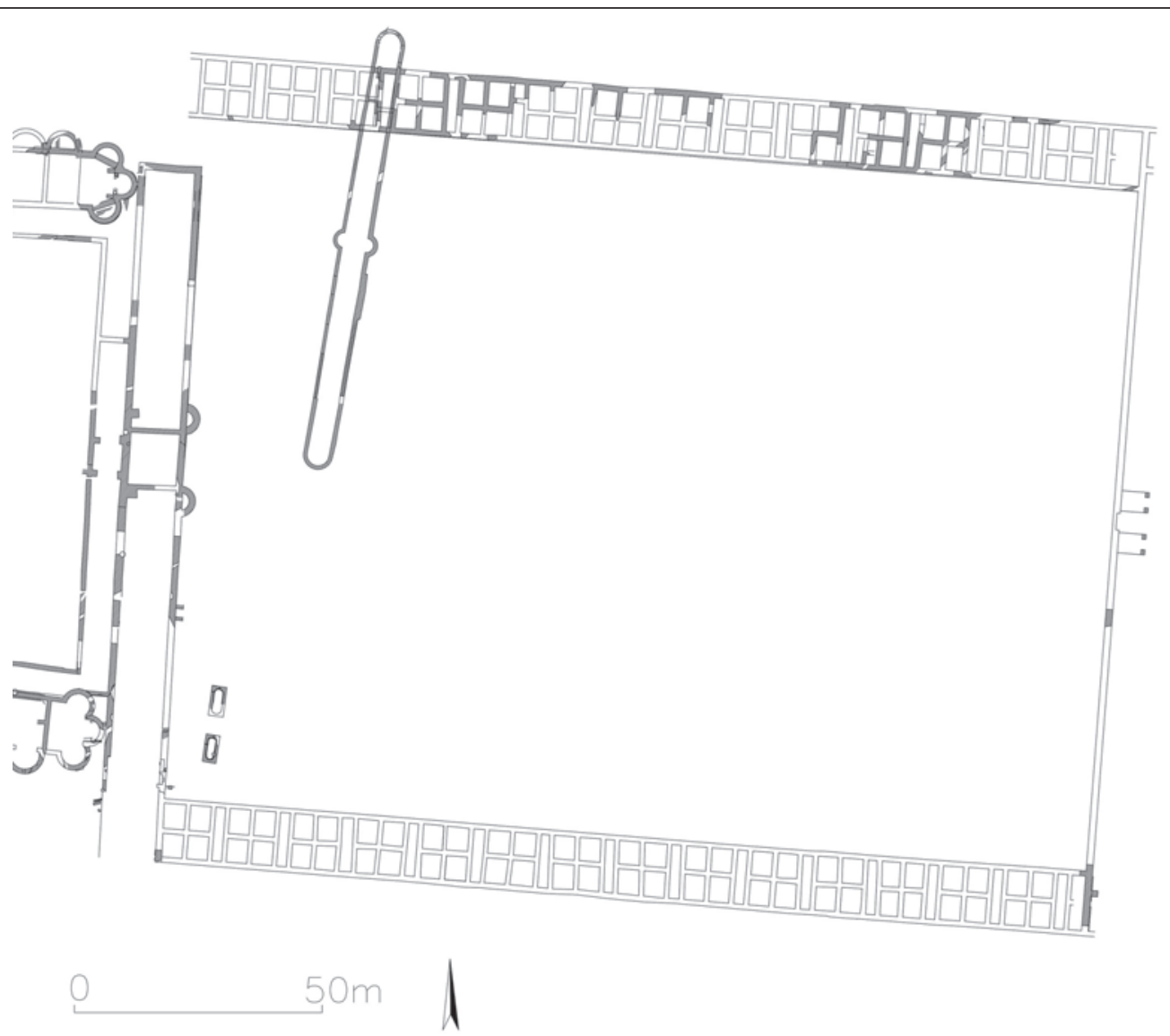

Figura 8.- Detalle de la superposición del cuerpo de servicio sobre el estanque inacabado.

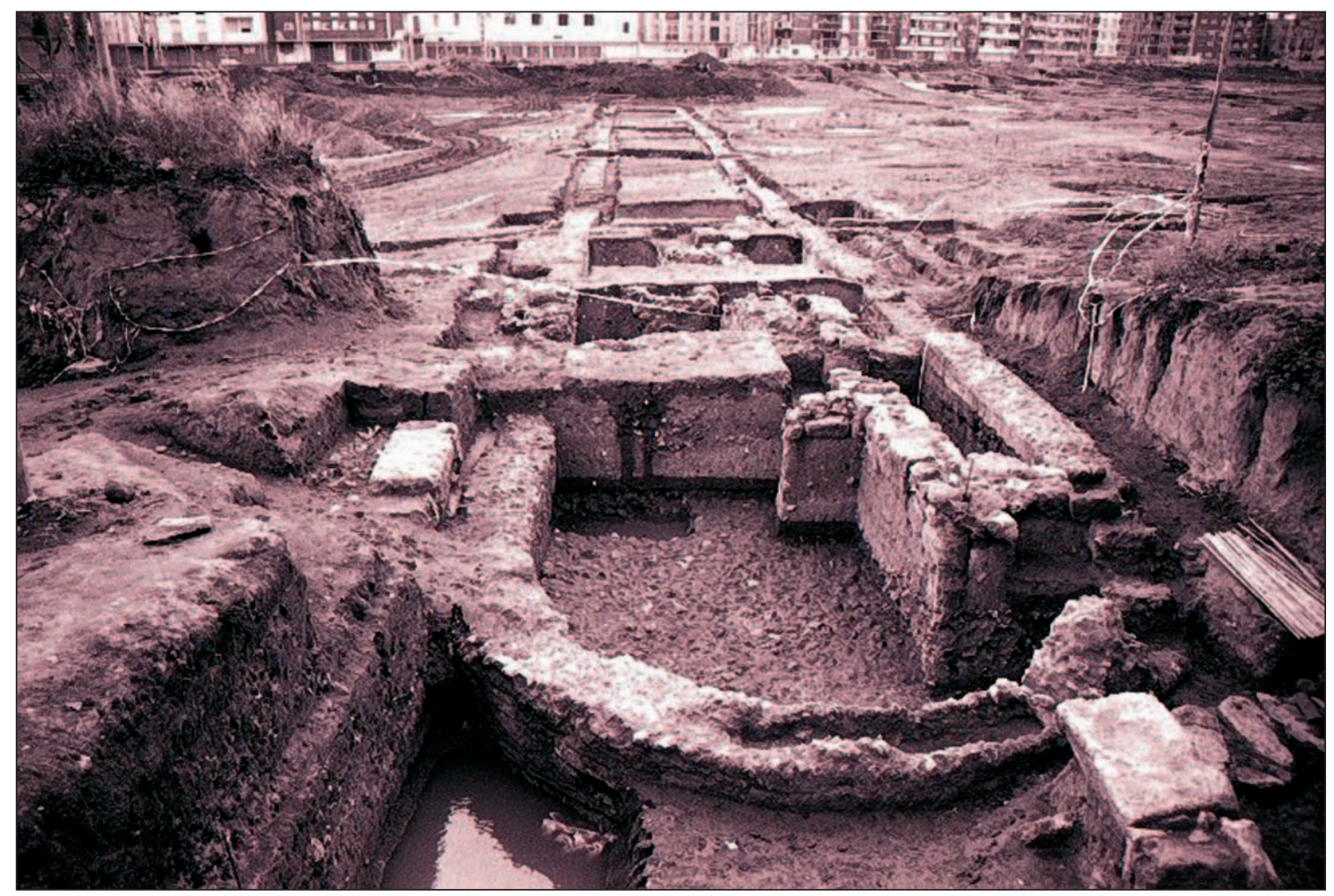

Figura 9.- Estanque inacabado y abandonado durante el proceso de obra (desde el norte). En primer plano se observa el rudus, sin vestigio alguno del pavimento final de sigininum. 
gitud por 4'5-4'8 m. de anchura, coronado con ábsides en los extremos y con dos pequeñas exedras, también absidadas, afrontadas en el centro. El juego ornamental que desarrollan todos estos elementos, deja bien claro que esta estructura se debe interpretar como un estanque ornamental y no con una de las estructuras de obra que, como se ha documentado en otras zonas del palacio (Hidalgo, 1997, passim), eran abandonadas una vez concluido el edificio. Tanto el sistema constructivo como la propias características de la construcción -ya sean sus dimensiones, su planta, en la que el elemento curvo se hace tan presente como en el resto del palacio, etc.-, permiten también adscribir esta estructura al palacio y descartar su posible vinculación con la villa preexisten$\mathrm{te}^{14}$. Al mismo tiempo, que este estanque nunca se llegó a concluir, queda perfectamente evidenciado por el hecho de que nunca contó con un pavimento hidráulico. Se construyó hasta el rudus sobre el que debería haber descansado el opus signinum, que nunca se llegó a incorporar ${ }^{15}$. Así pues, a la vez que se construye el palacio esta estructura se abandona sin llegarse a concluir, se transforma el concepto y forma de la plaza que antecede la zona neurálgica del palacio, y sobre el estanque inconcluso se construye uno de los cuerpos de servicio que flanquean la gran plaza que da acceso al palacio, fruto de la nueva concepción del conjunto.

Otro caso de replanteo y reforma del proyecto durante la propia obra es el relativo a la puerta abierta en el criptopórtico, en su lienzo perimetral (Hidalgo et alii, 1996, 11-42) (fig. 10). Esta puerta se construyó con la vocación de comunicar el criptopórtico con el espacio que lo rodea -en un tramo que no coincide con ninguna de las construcciones perimetrales conectadas con el pórtico en sigma-, para constituir muy probablemente un acceso de servicio. Con total seguridad, la puerta

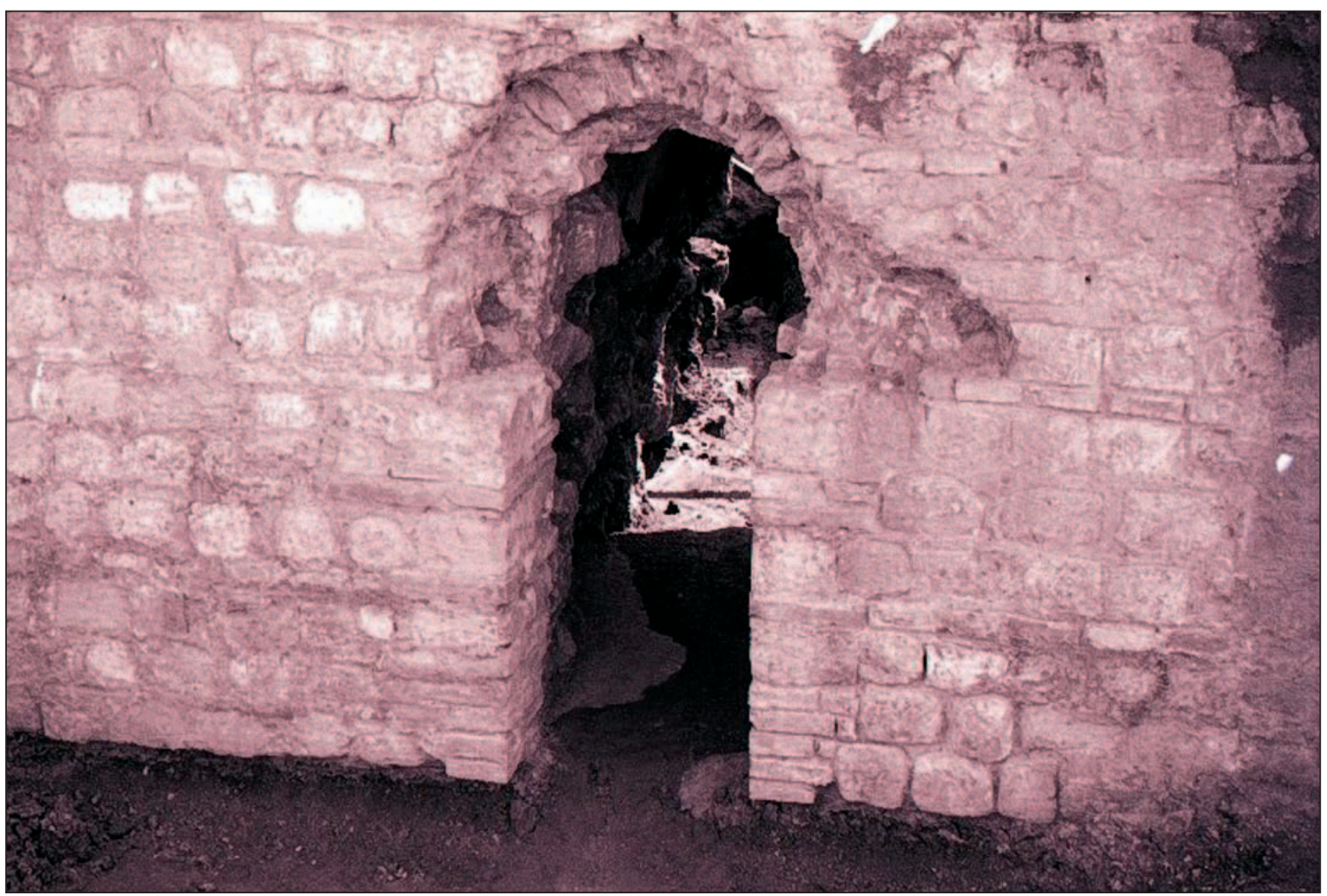

Figura 10.- Puerta del muro perimetral del criptopórtico.

14 Sobre la villa altoimperial véase especialmente Hidalgo, 1996, 24-28 y Moreno, 1997.

15 En relación con la ausencia de acabado final se debe descartar la posibilidad de que hubiera sido saqueado, pues no hay huella alguna de expolio y, sobre todo, porque, por sus propias características más que presumibles, esto es, el hecho de estar conformado por un sencillo pavimento de signinum, como es norma en tales construcciones, no habría supuesto de ninguna manera un bien preciado de cara a su reutilización, habida cuenta además que los muros del estanque no fueron en ningún tramo saqueados. 
se construyó a la vez que se llevaba a cabo el criptopórtico y, de hecho, el muro que lo conforma se interrumpe en este tramo, dejando un vano a partir del cual se debía materializar el tránsito en un momento constructivo posterior, dentro del propio proceso original de la obra. No obstante, como la excavación dejó perfectamente claro, como consecuencia de un cambio en el proyecto original este pasillo nunca llegó a materializarse y, por el contrario, el vano se cegó y tanto el interior del muro del criptopórtico como la zona trasera quedaron colmatados por los habituales rellenos constructivos.

Así pues, desgraciadamente, a partir de lo que hasta nosotros llegó de la sala triabsidada de Cercadilla, hoy completamente destruida, es imposible identificar con certeza cómo pudo ser su imagen final. Pero creo importante tener presente la idea de que, ante las dificultades que conlleva vincular los muros aquí analizados y dispuestos en su interior al sistema de sujeción de la cubierta de la estancia, y siempre partiendo de la premisa inequívoca de que todas las estructuras del edificio corresponden al proceso de edificación original del palacio, quizás durante el propio proceso de obra el edificio se pudo transformar, limitándose sus dimensiones y adaptándose a un modelo poco difundido pero sí muy vinculado a la arquitectura palatina de época tetrárquica.

\section{Bibliografía:}

BALDINI LIPPOLIS, I. (2001): La domus tardoantica. Forme e rappresentazioni dello spazio domestico nelle città del Mediterraneo, Bolonia.

BALMELLE, C. (2001): Les demeures aristocratiques d'Aquitaine: societé et culture de l'Antiquité tardive dans le Sud-Ouest de la Gaule, Burdeos.

BEK, L. (1983): “Questiones Convivales. The Idea of the Triclinium and the Staging of Convival Ceremony from Rome to Byzantium", Analecta Romana Istituti Danici 12, 81-107.

BETTINI, S. (1955): "Il castello di Maschattà in Transgiordania nell' ambito dell" "arte di potenza" tardoantica", Anthemom. Scritti di Archeologia e di Antichità Classiche in Onore di Carlo Anti, Floencia, 321-366.

BOWDEN, W. y HODGES, R. (2011 eds.): Butrint 3. Excavations at the Triconch Palace. Oxford.
CAMBI, N. (1984): "Triconch churches on the eastern Adriatic", X Congres. Int. d'Archeologia Chretienne, II, Vaticano, 45-54.

CARANDINI, A.; RICI, A. y VOS, M. DE (1982): Filosofiana: La villa di Piazza Armerina. Imagine di un aristocratico romano al tempo di Costantino. Palermo.

DEICHMANN, F.W. (1959): "Cella Trichora", Reallexikon für Antike und Christentum, Stuttgart, II, co. 944 y ss.

DOWNEY, S.B. (1993): "The Palace of the Dux Ripae at Dura-Europos and "Palatial" Architecture of Late Antiquity", Eius Virtutis Studiosi: Classical and Post-Classical Studies in Memory of Frank Edward Brown (1908-1988), Hannover, 183-200.

DUVAL, N. (1987): "Existe-t-il une «structure palatiale» propre à l'Antiquité tardive?, Le système palatial en Orient, Grèce et à Rome, Strasbourg, 463490.

DUVAL, N. y CINTAS, J. (1976): "Le martyrium de Cincari et les martyria triconques y tétraconques en Afrique", MEFR 88, 853-927.

EBERT, F. (1864): s.v. “Trichorum”, RE, Stuttgartt, v. VII Ai, col. 91.

FERNÁNDEZ CASTRO, M.C. (1982): Villas romanas en España, Madrid.

FUERTES, Ma . C. e HIDALGO, R. (2005): Guía Arqueológica de Cercadilla. Córdoba, Sevilla.

GROSSMANN, P. (1992): “The triconchoi in Early Christian Churches of Egypt and their origines in the architecture of Classical Rome", Roma $e$ l'Egitto nell'architettura classica (Atti del I Congresso Internazionale italo-egiziano), Roma, 182-190.

HIDALGO, R. (1996): Espacio público y espacio privado en el conjunto palatino de Cercadilla (Córdoba): el aula central y la termas, Sevilla.

HIDALGO, R. (1997): El complejo palatino de Cercadilla en Córdoba. Tesis doctoral inédita, Universidad de Córdoba.

HIDALGO, R. (1998): El triclinium triconque del palatium de Córdoba", AAC 9, 273-300.

HIDAlGO, R. (2004): "El Palatium”, en: Dupré, X. (ed.) Las capitales provinciales de Hispania, I. Córdoba. Colonia Patricia Corduba, Roma, 95104.

HIDALGO, R. (2007): "La puerta del Palatium de Cordvba". Romula 6, 143-172. 
HIDALGO, R. (2010): "La ciudad dentro de la ciudad: la tutela y conservación del Patrimonio Arqueológico en el ámbito urbano en Andalucía", en: Hidalgo, R. (ed.) La ciudad dentro de la ciudad. La tutela y conservación del Patrimonio Arqueológico en ámbito urbano, Sevilla, 5-20.

HIDALGO, R. y VENTURA, A. (1994): "Sobre la cronología e interpretación del palacio de Cercadilla en Corduba”, Chiron 24, 221-240.

HIDALGO, R. et alii (1993): "Excavación arqueológica en el yacimiento de Cercadilla. Campaña de 1993”, Anuario Arqueología de Andalucía, III/Actividades de Urgencia, Sevilla, 132-148.

HIDALGO, R. et alii (1996): El criptopórtico de Cercadilla: arquitectura y secuencia estratigráfi$c a$, Sevilla.

LAVIN, I. (1962): "The House of the Lord: Aspects of the Role of Palace triclinia in the architecture of Late Antiquity in the Early Middle Age", Art Bulletin 44, 1-27.

MORENO, M. (1997): La villa altoimperial de Cercadilla (Córdoba): análisis arqueológico, Sevilla.

MORVILLEZ, E. (1995): "Les sales de réception triconques dans l'architecture domestique de l'Antiquité tardive en Occident", Histoire de l'Art, 31, octubre, 15-26.
POINNSOT, C. (1958): Les ruines de Dougga, Túnez.

RIZZARDI, C. (1996): "I mosaici del triclinio del palatium di Teodorico a Ravenna", en: Guidobaldi, F. y Guiglia guidobaldi, A. (eds.) Atti del III Colloquio dell'Associazione italiana per lo studio e la conservazione del mosaico, Bordighera, 353-361.

ROSSITER, J. (1991): Convivium and villa in Late Antiquity", en: Slater, W.J. (ed.) Dinning in Classical Context, Ann Arbor, 199-214.

SCAGLIARINI CORLÀITA, D, (2003): "Domus, villae, palatia. Convergenze e divergenze nelle tipologie architettoniche, en: Ortalli, J. y Heinzelmann, M. (eds.), Abitare in città. La Cisalpina tra impero e medioevo, Palilia 12, Wiesbaden, 153-172.

SETIS, S. (1975): “Per l'interpretazione di Piazza Armerina", MEFRA 87, 873-994.

SFAMENI, C. (2006): Ville residenziali nell'Italia tardoantica, Bari.

VERZONE, P. (2011): Palazzi e domus. Dalla tetrarchia al VII secolo, Roma.

YEGÜL, F. (1992): Baths and Bathing in Classical Antiquity, New York. 